\section{La vie révélée du follicule de cheveu humain}

Bruno A. Bernard

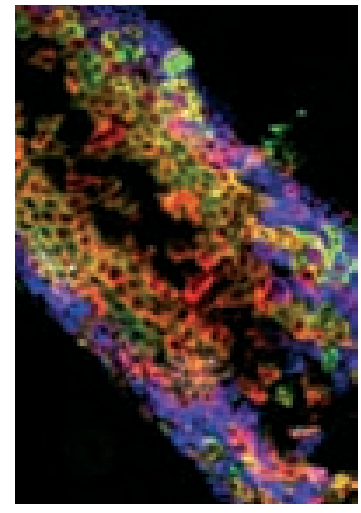

L’Oréal Recherche, Centre C. Zviak, 90 , rue du Général Roguet, 92110 Clichy, France. bbernard@rd.Ioreal.com

morphogènes dont la nature commence à être connue, mais dont la chorégraphie spatiotemporelle est encore à déchiffrer, en particulier chez l'homme. En effet, si les processus morphogénétiques de la vibrisse et du poil de souris commencent à être bien caractérisés $[3,5]$, ce n'est que par analogie que l'on peut proposer un scénario pour le follicule de cheveu humain.

La mise en place des placodes et le motif d'implantation des cheveux seraient contrôlés par une série de facteurs initiateurs (représentants des familles Wnt/Frz, FGF/ FGFR2, TGF $\beta 2 / T G F \beta R I I, E D A / E D A R$, Delta-1/Notch-1) ou inhibiteurs (BMP2/4, activins, Delta-1/Notch-1) [3, $5,6]$, et de régulateurs transcriptionnels de la famille des protéines homeobox [7] ; leurs interactions aboutiraient à la formation de zones discrètes, susceptibles de former des follicules [8], et à la sélection des cellules destinées à former la partie épithéliale du follicule [9]. Ainsi, du côté dermique, l'inhibiteur de BMP Noggin renforcerait les zones d'induction du follicule. Au cours de la phase d'invagination dans le mésoderme suivant la formation de la placode, les cellules épithéliales émettent des messagers, parmi lesquels figurent des représentants de la famille Wnt, le PDGF-A et shh, qui vont provoquer l'agrégation de fibroblastes du derme en une papille dermique en regard du bourgeon primaire. Les deux éléments majeurs du follicule pileux, une partie épithéliale et une partie dermique, sont alors en place. De façon intéressante, les facteurs Wnt, à travers leurs récepteurs frz et la voie de la $\beta$-caténine/LEF-1, peuvent contrôler l'expression de shh et des 
BMP [10], soulignant ainsi l'intrication des réseaux de signalisation. La voie Wnt peut également activer des gènes à homeobox, ainsi que certains gènes de kératines spécifiques de la tige pilaire. Après ce processus de morphogenèse, comparable dans sa complexité à celui de la dent ou de la plume [2], le follicule subit, chez l'adulte, des modifications cycliques présentant pour certaines des caractéristiques réminiscentes des processus embryonnaires qui ont abouti à sa formation.

\section{Structure du follicule pileux}

L'analyse histologique et immunohistologique montre que le follicule pileux est formé de compartiments parfaitement individualisés, les uns d'origine dermique (gaine conjonctive et papille dermique), les autres de nature épithéliale (gaine épithéliale externe, gaine interne, tige pilaire et glande sébacée) (Figure 1).

La gaine conjonctive, synthétisée par des fibroblastes, est surtout une matrice extracellulaire formée de collagènes de types I et III, ainsi que de protéoglycanes. Traversée, dans le tiers inférieur, par un fin réseau de capillaires sanguins, elle se prolonge à la base du follicule par la papille dermique, véritable agrégat de matrice extracellulaire. La papille dermique est synthé-

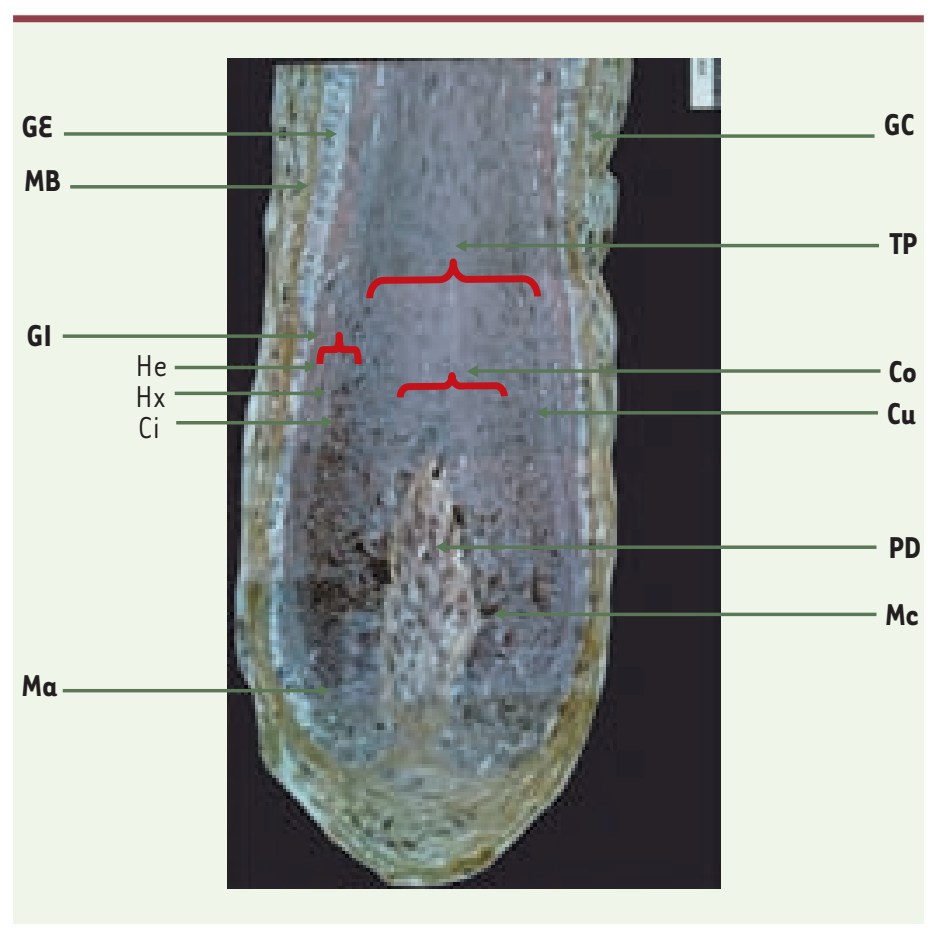

Figure 1. Compartiments du bulbe pilaire. $\mathrm{Ci}$ : cuticule de la gaine interne; $\mathrm{Co}$ : cortex; $\mathrm{Cu}$ : cuticule; GC: gaine conjonctive; GE: gaine externe; GI: gaine interne; He : couche de Henle ; Hu : couche de Huxley; Ma : matrice; MB : membrane basale; Mc : mélanocyte ; PD : papille dermique ; TP : tige pilaire. tisée par des fibroblastes particuliers, exprimant des marqueurs spécifiques tels que la phosphatase alcaline, la protéine anti-apoptotique $\mathrm{Bcl}-2$ et la cyclooxygénase de type 1 , responsable de la synthèse locale de prostaglandine $\varepsilon 2$.

Une membrane basale, composée de collagène de type IV, de laminine de type 1 (et 5), de fibronectine et d'héparane-sulfate protéoglycanes, sépare le compartiment dermique du compartiment épithélial. Le profil d'expression de la laminine 5 (ligand de l'intégrine $\alpha 6 \beta 4$ ) est toutefois interrompue à la périphérie du bulbe [11], soulignant ainsi une régionalisation de la jonction dermo-épidermique.

Le compartiment épithélial peut, quant à lui, être séparé en quatre domaines distincts. À la base du follicule et entourant la papille dermique se trouve la matrice, siège d'une intense activité mitotique. Cette matrice produit ainsi, avec des programmes de différenciation spécifiques, les trois grands domaines concentriques que sont la gaine externe, la gaine interne et la tige pilaire: la gaine interne est ellemême formée de trois couches concentriques (couche de Henle, couche de Huxley et cuticule), tout comme la tige pilaire (cuticule, cortex et médulla). Chacun de ces domaines expriment des marqueurs spécifiques : Ia gaine externe, par exemple, exprime un ensemble de kératines bien plus complexe que celui de l'épiderme, puisqu'il inclut les kératines $\mathrm{K} 5, \mathrm{~K} 6, \mathrm{~K} 8, \mathrm{Kl} 4, \mathrm{~K} 16, \mathrm{~K} 17, \mathrm{~K} 18$, et $\mathrm{K} 19$, certaines d'entre elles ayant une expression de type mosaïque (Figure 2). La gaine interne, dont le programme de différenciation est partiellement sous le contrôle du facteur de transcription GATA-3, exprime la trichohyaline, les transglutaminases 1 et 5 et la kératine hK6irs. Quant à la tige pilaire, sous le contrôle partiel du facteur de transcription LEF-1/CTF, elle exprime de façon très spécifique la transglutaminase 3 [12], ainsi qu'une quinzaine de kératines spécifiques, 9 acides et 6 basiques, réparties de façon très précise dans la cuticule, la zone kératogène ou le cortex [13]. Plus d'une centaine de protéines, plus ou moins enrichies en cystéine, sont exprimées en association avec les kératines (grande famille des KAP). Dans la partie supérieure du follicule se trouve la glande sébacée, formée de kératinocytes particuliers, les sébocytes (spécifiquement marqués par les kératines $\mathrm{K} 4$ et K15), responsables de la synthèse du sébum. La partie supérieure de la gaine externe est également caractérisée par la présence de cellules de Langerhans, véritables sentinelles du système immunitaire, et de cellules de Merkel (spécifiquement marqués par la kératine K20), remplies de neuropeptides et dont le rôle est encore méconnu.

Comme on peut le voir, la structure du follicule pileux est extrêmement complexe, avec des compartiments concentriques organisés autour d'un axe de symétrie. Toutefois, une des caractéristiques du cheveu humain est la diversité de sa forme (raide, bouclée ou crépue) : en fait, la courbure du cheveu est l'expression d'une précontrainte interne à la fibre, 
résultant d'une asymétrie touchant les programmes de différenciation des différents compartiments du follicule, au niveau du bulbe [14]. Ainsi, le follicule associé à un cheveu bouclé est luimême courbé, et caractérisé par une rétrocourbure au niveau du bulbe. Les cellules de la matrice prolifèrent de façon plus active du côté convexe que du côté concave de la rétrocourbure. En revanche, les programmes de différenciation de la gaine externe, de la gaine interne et, pour une certaine part, de la tige (notamment de la cuticule), démarrent de façon plus précoce du côté concave que du côté convexe [14]. L'origine moléculaire de cette asymétrie du bulbe reste à ce jour inconnue.

Malgré cette complexité, l'organisation interne du follicule pileux est incroyablement stable, puisqu'elle est préservée lorsque des follicules disséqués à partir de biopsies de cuir chevelu sont mis en culture in vitro. Dans ces conditions, le follicule continue à produire de la fibre, à la vitesse de $0,3 \mathrm{~mm} / \mathrm{j}$. Des follicules ont ainsi été maintenus en survie pendant 45 jours, et des études par immunohistochimie [15] et diffraction des rayons $X$ [16] ont montré que l'organisation moléculaire de la fibre pilaire était conservée. Cela souligne le caractère unique du follicule pileux, à savoir son homéostasie, probablement assurée par un réseau com-

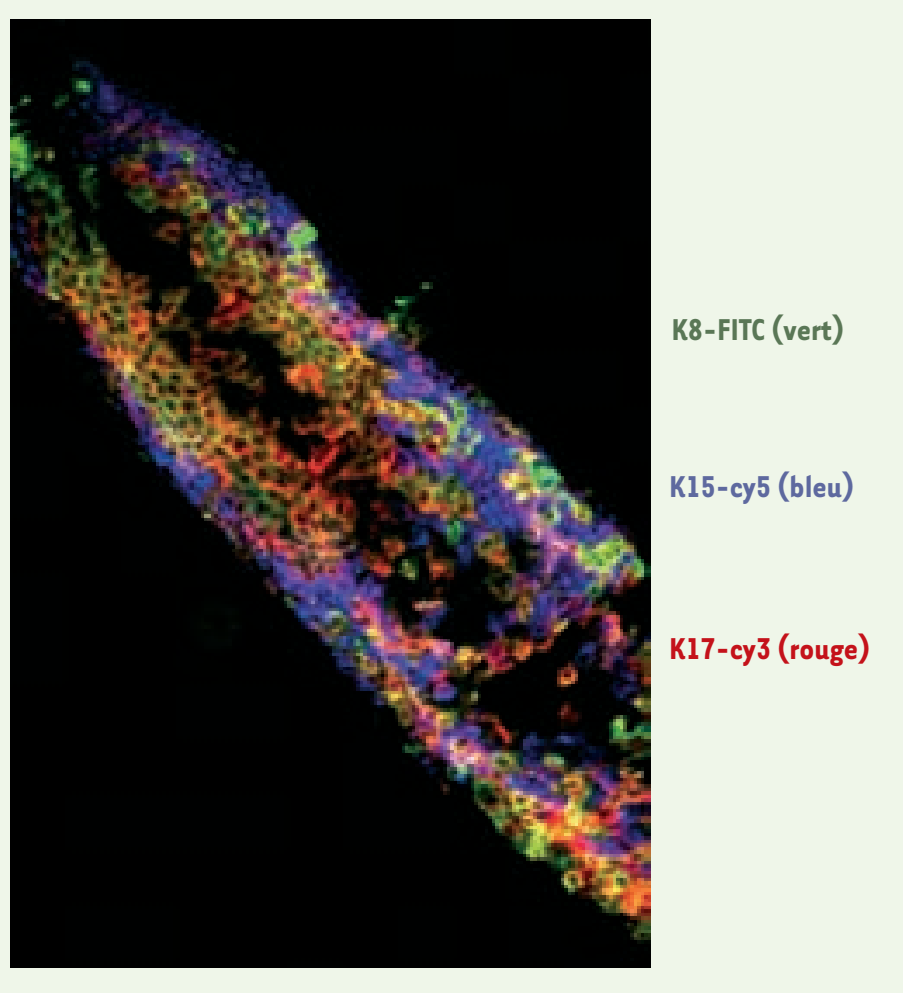

Figure 2. Mosaïcisme de l'expression des kératines dans la gaine externe. Marquage avec des anticorps spécifiques et analyse par microscopie confocale laser. En vert, kératine K8 (isothiocyanate de fluorescéine, FITC); en bleu, kératine K15 (cyanine Cy5); en rouge, kératine K17 (cyanine Cy3). Noter que la kératine $K 15$ décore de façon sélective certaines cellules, tandis que les kératines K8 et K17 semblent majoritairement co-exprimées. plexe d'interactions cellulaires, et par des boucles de contrôle autocrines et paracrines.

\section{Dynamique du cycle pilaire}

Un follicule pileux est en phase de production de tige pilaire (phase anagène), en involution (phase catagène) ou en phase de repos, avec chute du cheveu (phase télogène) [17]: la durée moyenne de chacune de ces phases est de 3 ans, 3 semaines et 3 mois, respectivement. Sur une chevelure normale, environ $85 \%$ des follicules sont en phase anagène, et $15 \%$ en phase télogène. À l'issue des phases catagène et télogène, la plupart des compartiments du follicule ont été dégradés par un processus actif d'apoptose, à l'exception notoire de la papille dermique. À la suite de la phase télogène, et par un processus de néo-morphogénèse, le follicule se régénère sur place à partir d'un réservoir de cellules pluripotentes, et entame une nouvelle phase anagène. Une phase de latence de quelques mois, la phase exogène [18], est parfois observée entre la phase télogène et la nouvelle phase anagène $[17,19]$. Ces phases successives constituent le cycle pilaire.

La technique du phototrichogramme a permis de montrer que le cheveu pousse à la vitesse de $0,3 \mathrm{~mm} / \mathrm{j}$ ) [20], que la durée des phases varie d'un individu à l'autre [17] et que la phase dont l'amplitude varie le plus au cours du temps est la phase anagène [17, 19]. De plus, si l'on considère un follicule pris individuellement, la durée de ses différentes phases varie d'un cycle à l'autre de façon apparemment alétoire, ce qui suggère l'absence d'influence du cycle $n$ sur le cycle $n+1$, et l'absence d'influence de la durée de la phase $i$ sur celle de la phase $i+1$ [21]. Enfin, le pourcentage des cheveux en phase anagène et télogène fluctue au cours du temps, autour d'une valeur moyenne [21]. De l'analyse de ces fluctuations et du caractère apparemment aléatoire de la durée des phases, il ressort que les transitions d'une phase à l'autre se produisent indépendamment pour chaque follicule, de façon non déterministe, après des intervalles de temps donnés, de façon stochastique, à partir d'une loi log-normale de distribution caractérisée par une moyenne et une variance pour chacune des phases [21].

Le comportement stochastique de chaque follicule assure la permanence de la chevelure, mais reflète aussi la multiplicité des acteurs susceptibles de moduler son activité : facteurs de croissance (IGF-1, HGF, FGF-2, KGF...), hormones (androgènes et œstrogènes, mais aussi vitamine $D$ et tri-iodothyronine [22-25]), cytokines (comme I'IL-1 $\alpha$ [26]). Le poids relatif de tous ces facteurs est 
lui-même modulé au niveau génétique, à travers des polymorphismes [27] et des mutations faux-sens ou non-sens [28]: en effet, certaines personnes perdent leurs cheveux, et d'autres non. Cette perte de cheveu touche les hommes et les femmes, mais avec des profils différents: chez l'homme, la chute se localise surtout sur les tempes et le vertex, tandis que, chez la femme, la chute est diffuse. Si l'origine de la chute des cheveux n'a pas encore été élucidée, elle se caractérise néanmoins par une augmentation progressive de l'hétérogénéité du diamètre des cheveux, et par l'apparition de signes péripilaires, signature d'une phase inflammatoire provisoire qui semble précéder l'épaississement de la gaine conjonctive et la miniaturisation du follicule [29].

\section{Renouvellement du follicule}

À chaque cycle pilaire, lors de la transition télogène-anagène, la structure folliculaire est entièrement régénérée, tant au niveau des compartiments épithéliaux que du compartiment pigmentaire, responsable de la couleur du cheveu. La papille, quant à elle, se remet à synthétiser de façon active la matrice extracellulaire, perdue lors des phases catagène et télogène.

Contrairement à ce qui peut être observé chez la souris, la surexpression de l'intégrine $\beta 1$ [11] et l'expression de la kératine 19 (K19) [30] ont permis d'identifier deux zones enrichies en cellules «souches » épithéliales, situées dans la partie proximale du follicule, au-dessus du bulbe, et dans sa partie distale, sous la glande sébacée. L'existence de ces deux groupes de cellules souches pluripotentes est corroborée par le fait que de l'épiderme interfolliculaire peut être reconstruit in vitro à partir de ces deux zones [31]. Durant la phase catagène, l'anneau inférieur de cellules $\mathrm{K} 9^{+}$n'est pas détruit, et migre avec la colonne épithéliale en régression pour finalement fusionner avec l'anneau supérieur, dans la capsule télogène. Lors du redémarrage d'un nouveau cycle, une fraction des cellules $\mathrm{K} 9^{+}$redescend avec le follicule nouvellement formé, pour redonner l'anneau inférieur. Cette follicule. population de cellules $\mathrm{K}^{19^{+}}$subit donc, au cours des cycles successifs, un processus de fusion-séparation [30] qui maintient deux zones de cellules pluripotentes [32, 33] (Figure 3A).

Outre sa croissance et son renouvellement cyclique, le cheveu humain est caractérisé par la présence de grains de mélanine dans son cortex, responsable de sa couleur. Cette mélanine est produite par des mélanocytes actifs, localisés dans le bulbe anagène à l'apex de la papille dermique, où ils forment l'unité de pigmentation. Une autre population de mélanocytes, quiescents et inactifs quant à la mélanogenèse, réside dans la région supérieure de la gaine externe du follicule, sous l'abouchement de la glande sébacée. À chaque cycle, l'unité de pigmentation détruite au cours de la transition anagène-catagène est régénérée lors de la phase de néomorphogenèse. En fait, les mélanocytes quiescents de la gaine externe forment un réservoir à partir duquel, à chaque transition télogène-anagène, une sous-population est recrutée pour repeupler le bulbe du follicule nouvellement formé et régénérer une unité de pigmentation, tandis qu'une

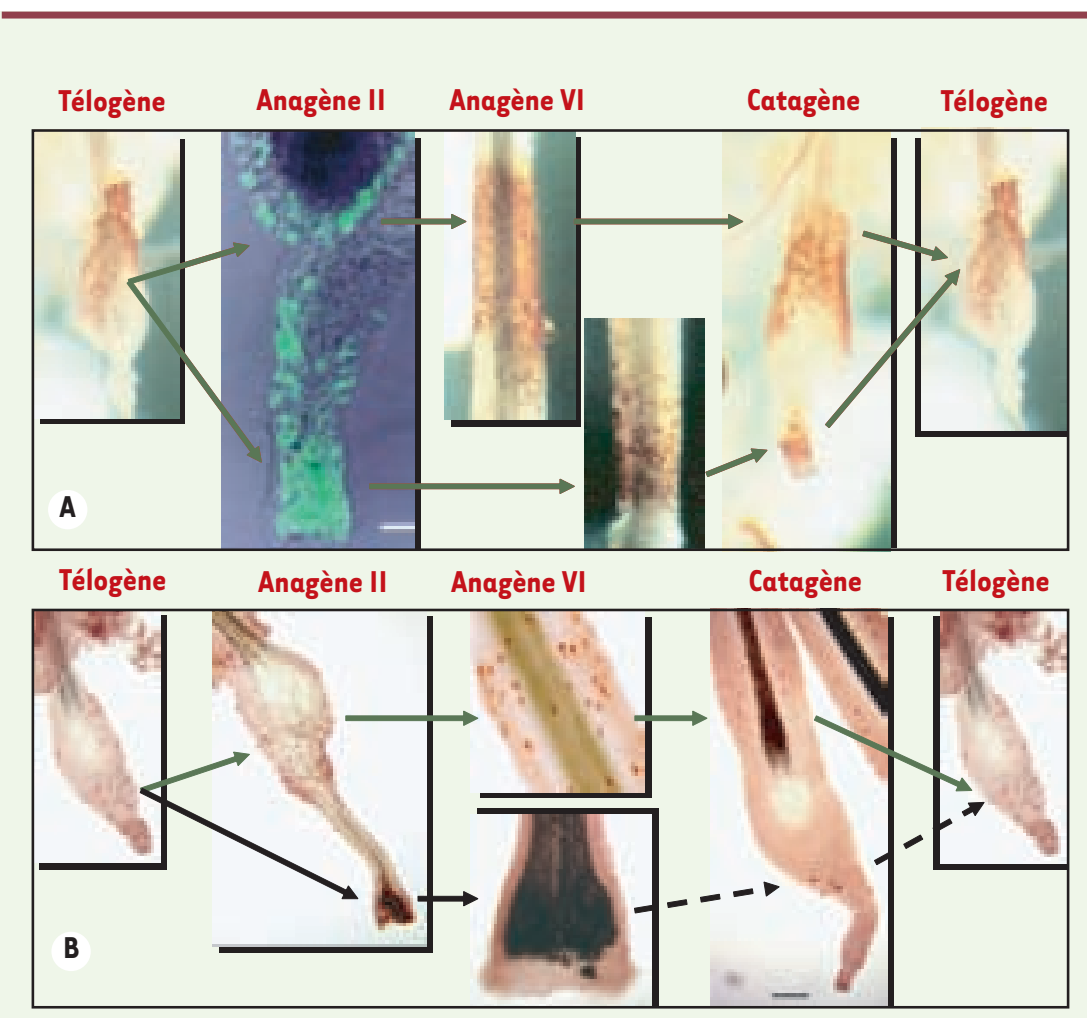

Figure 3. Dynamique des réservoirs de cellules souches épithéliales et de mélanocytes progéniteurs au cours du cycle pilaire. Les cellules souches épithéliales sont identifiées par l'expression de la kératine 19, tandis que les mélanocytes sont identifiés par l'expression de la protéine prémélanosomale Pmel-17. A. Réservoirs de cellules souches épithéliales: noter les deux compartiments, bien individualisés en phase anagène, qui fusionnent au cours des phase catagène et télogène pour se re-séparer à l'initiation du cycle suivant (anagène II) : ce comportement est à l'origine de l'hypothèse «fusion/séparation » [24]. B. Réservoir de mélanocytes progéniteurs: noter les deux populations mélanocytaires qui s'individualisent lors de l'initiation d'un nouveau cycle (anagène II). La population qui reste dans la partie distale représente le réservoir de mélanocytes progéniteurs, celle qui migre avec le bulbe est l'unité de pigmentation du nouveau 
autre sous-population va rester dans la partie supérieure de la gaine externe [34] (Figure 3B). De façon remarquable, seuls les mélanocytes du bulbe nouvellement formé prolifèrent, de façon transitoire, puis se remettent à exprimer les marqueurs de mélanogenèse (tyrosinase et TRP-1).

\section{Processus de blanchissement du cheveu}

Jusque récemment, le blanchissement chronologique avait été associé à plusieurs causes possibles, parmi lesquelles l'arrêt de synthèse de mélanines et l'arrêt de transfert des mélanosomes. En fait, le processus de blanchissement est dû à une décroissance progressive du nombre des mélanocytes, tant au niveau de l'unité de pigmentation que du réservoir [35]. Lorsque le nombre de mélanocytes actifs atteint un seuil limite, la quantité de mélanine synthétisée et transférée à la tige pilaire en croissance n'est plus suffisante pour que le cheveu soit perçu pigmenté, il est donc perçu blanc; à terme, les mélanocytes disparaissent totalement de l'unité de pigmentation et du réservoir. Ce processus de déclin n'affecte, de façon très spécifique, que le follicule pileux, puisque l'infundibulum et l'épiderme interfolliculaire adjacent ne sont pas touchés. Si elle reste obscure, la raison de cette décroissance progressive semble liée à l'absence d'expression de la dopachrome tautomérase (ou TRP-2) dans les mélanocytes folliculaires, par opposition à la situation observée dans les mélanocytes de l'infundibulum et épidermiques, qui l'expriment de façon constitutive [36]. II est possible que TRP-2 ait une fonction de protection vis-à-vis du mélanocyte, et que son absence d'expression induise une sensibilité particulière du mélanocyte folliculaire aux agressions de type oxydatif, notamment.

\section{Conclusions}

Le follicule de cheveu humain apparaît comme un organe qui, de façon unique dans l'organisme humain, se renouvelle de façon cyclique, autonome et stochastique. Pour assurer ce renouvellement, le follicule pileux possède ses propres réservoirs de cellules épithéliales pluripotentes et de mélanocytes progéniteurs. Bien que des processus de recrutement et de sélection semblent toucher ces deux types de réservoirs à la transition télogène-anagène, ces processus sont probablement indépendants, puisqu'au cours du vieillissement chronologique, des cheveux peuvent continuer à pousser vigoureusement tout en ayant blanchi, alors que des cheveux peuvent se miniaturiser tout en restant pigmentés. En ce qui concerne le blanchissement du cheveu, son caractère progressif laisse ouverte la possibilité de le prévenir, en empêchant ou en ralentissant la disparition des mélanocytes du réservoir, ou de l'inverser, en réactivant ces mêmes mélanocytes du réservoir. $\diamond$

\section{SUMMARY}

\section{The revealed life of human hair follicle}

The human hair follicle is a unique appendage which results from epithelio-mesenchymal interactions initiated around the $3^{\text {rd }}$ month of development. This appendage has a very complex structure, with a dermal compartment and an epithelial compartment. The dermal compartment comprises the connective tissue sheath and the dermal papilla, both of which are irrigated by microvessels. The epithelial compartment is made of highly replicating matrix cells giving rise to three concentrical domains, namely the outer root sheath, the inner root sheath and the hair shaft. The pigmentation unit, responsible for hair color, is made of fully active melanocytes located on top of the dermal papilla. Altogether a hair follicle contains more than 20 different cell types, engaged in different differentiation pathways and/or interacting with each other. This complex appendage has a unique behavior in mammals since, after a hair production phase, it involutes in place before entering a resting phase after which it renews itself under a cyclical but stochastic way, out of a double reservoir of pluripotent stem cells able to also regenerate epidermis. For yet unknown reasons, this well ordered process can be disturbed, provoking alopecia. The pigmentation unit also renews itself under a cyclical way, out of a melanocyte progenitor reservoir which progressively declines with time, provoking the hair whitening process. Finally, the shape of the hair shaft is programmed from the bulb. What makes this appendage unique and fascinating is its high degree of autonomy, its incredibly complex though stable structure, the number of different cell types interacting under an equilibrated way and its potential of regeneration. It represents a true paradigm of tissue homeostasis, exemplifying in a small living cylinder all the fundamental laws of cell-cell and tissue interactions. This life is revealed in this short synthesis. $\diamond$

\section{ABRÉVIATIONS}

BMP2/4 : bone morphoprotein 2/4

EDA/EDAR : ectodysplasin/EDA receptor

FGF/FGFR : fibrobast growth factor/FGF receptor

Frz : frizzled (récepteurs des ligands de la famille Wnt)

GATA-3 : GATA binding protein 3

HGF : hepatocyte growth factor

hK6irs: keratin specific to the inner root sheath

IGF : insulin like growth factor

IL- $1 \alpha$ : interleukine- $1 \alpha$

KAP : keratin associated proteins

KGF : keratinocyte growth factor

LEF-1 : lymphoid enhancer factor 1

PDGF-A : platelet-derived growth factor-A

Pmel-17 : potentially malignant epithelial lesion 17

Shh : sonic-hedge-hog

TGF/TGFR : transforming growth factor/TGF receptor

TRP-1 : tyrosinase-related protein-1

Wnt : wingless homolog (Drosophila) 


\section{RÉFÉRENCES}

1. Olivera-Martinez I, Viallet JP, Michon F, et al. The different steps of skin formation in vertebrates. Int J Dev Biol $2004 ; 48: 137-48$.

2. Pispa J, Thesleff I. Mechanisms of ectodermal organogenesis. Dev Biol $2003 ; 262: 195-205$.

3. Milar SE. Molecular mechanisms regulating hair follicle development. J Invest Dermatol 2002 ; $118: 216-25$

4. Holbrook KA, Smith LT, Kapla ED, et al. Expression of morphogens during human follicle development in vivo and a model for studying follicle morphogenesis in vitro. J Invest Dermatol $1993 ; 101: 39 s-49 s$.

5. Schmidt-Ullrich R, Paus R. Molecular principles of hair follicle induction and morphogenesis. Bioessays $2005 ; 27: 247-61$.

6. Reddy ST, AndI T, Lu MM, et al. Expression of Frizzled genes in developing and postnatal hair follicles. J Invest Dermatol $2004 ; 123: 275-82$.

7. Awgulewitsch A. Hox in hair growth and development. Naturwissenschaften 2003 ; $90: 193-211$.

8. Mooney JR, Nagorcka BN. Spatial patterns produced by a reaction-diffusion system in primary hair follicles. J Theor Biol 1985; 115 : 299-317.

9. Botchkarev VA, Sharov AA. BMP signaling in the control of skin development and hair follicle growth. Differentiation $2004 ; 72: 512-26$

10. van Steensel MAM, va Geel M, Steijlen PM. The molecular basis of hair growth. Eur J Dermatol $2001 ; 11: 348-52$.

11. Commo S, Bernard B A. The distribution of alpha 2 beta 1 , alpha 3 beta 1 and alpha 6 beta 4integrins identifies distinct subpopulations of basal keratinocytes in the outer root sheath of the human anagen hair follicle. Cell Mol Life Sci 1997 ; 5 : 466-71.

12. Thibaut $S$, Candi $\varepsilon$, Pietroni $V$, et al. Transglutaminsae 5 expression in human hair follicle. J Invest Dermatol $2005 ; 125: 581-5$.

13. Langbein L, Schweizer J. Keratins of the human hair follicle. Int Rev Cytol 2005 ; 243 : 1-78.

14. Thibaut $S$, Gaillard 0 , Bouhanna $P$, et al. Human hair shape is programmed from the bulb. BrJ Dermatol $2005 ; 152: 632-8$.

15. Thibaut $\mathrm{S}$, Collin C, Langbein L, et al. Hair keratin pattern in human hair follicles in vitro. Exp Dermatol $2003 ; 12: 160-4$.

16. Baltenneck F, Bernard BA, Garson JC, et al. Study of the keratinization process in human hair follicle by X-ray microdiffraction. Cell Mol Biol $2000 ; 46$ : 1017-24.

17. Courtois M, Loussouarn G, Hourseau C, Grollier JF. Hair cycle and alopecia. Skin Pharmacol $1994 ; 7: 84-9$.

18. Stenn K, Parimoo S, Prouty SM. Growth of the hair follicle : a cycling and regenerating biological system. In : Chuong CM, ed. Molecular basis of epithelial appendage morphogenesis. Austin : Landes Publishers, 1998 : 111-30.

19. Courtois M, Loussouarn G, Hourseau C, Grollier JF. Ageing and hair cycles. Br J Dermatol 1995 ; $132: 86-93$.

20. Loussouarn G. African hair growth parameters. Br J Dermatol 2001 ; $145: 294-7$.

21. Halloy J, Bernard BA, Loussouarn G, Goldbeter A. Modeling the dynamics of human hair cycles by a follicular automaton. Proc Natl Acad Sci USA $2000 ; 97: 8328-33$.

22. Billoni N, Gautier B, Mahé YF, Bernard BA. Expression of retinopid nuclear receptor superfamily members in human hair follicles and its implication in hair growth. Acta Derm Venereol (Stockh) $1997 ; 77: 350-5$.
23. Gerst C, Dalko M, Pichaud P, et al. Type-1 steroid 5 alpha-reductase is functionally active in the hair follicle as evidenced by new selective inhibitors of either type-1 or type- 2 human steroid 5 alpha-reductase. Exp Dermatol 2002 ; 11 h 52-8.

24. Thornton MJ, Taylor AH, Mulligan K, et al. Oestrogen receptor beta is the predominant oestrogen in human hair scalp skin. Exp Dermatol 2003; 12 : 181-90.

25. Billoni N, Buan B, Gautier B, et al. Thyroid hormone receptor bl is expressed in the human hair follicle. BrJ Dermatol $2000 ; 142: 645-52$.

26. Mahé $Y F$, Buan B, Billoni N, et al. Pro-inflammatory cytokine cascade in human plucked hair. Skin Pharmacol $1996 ; 9$ : 366-75.

27. Birch MP, Messenger AG. Genetic factors predispose to balding and nonbalding in men. Eur J Dermatol $2001 ; 11: 309-14$

28. Bernard BA. Hairless, il s'en est fallu d'un cheveu. Med Sci (Paris) 2002 ; $18: 276-80$.

29. Deloche $C$, de Lacharrière 0 , Mischiali $C$, et al. Histological features of peripilar signs associated with androgenetic alopecia. Arch Dermatol Res 2004 ; $295: 422-8$.

30. Commo S, Gaillard 0, Bernard BA. The human hair follicle contains two distinct $\mathrm{K} 19$ positive compartments in the outer root sheath : a unifying hypothesis for stem cell reservoir? Differentiation $2000 ; 66: 157-64$.

31. Lenoir MC, Bernard BA, Pautrat G, et al. Outer root sheath cells of human hair follicle are able to regenerate a fully differentiated epidermis in vitro. Dev Biol $1988 ; 130: 610-20$.

32. Panteleyev A, Jahoda CAB, Christiano AM. Hair follicle predetermination. J Cell Sci $2001 ; 114: 3419-31$.

33. Ghali L, Wong ST, Tidman N, et al. Epidermal and hair follicle progenitor cells express melanoma-associated chondroitin sulphate proteoglycan. J Invest Dermatol 2004 ; 122 : 433-42.

34. Commo S, Bernard BA. Melanocyte subpopulation turnover during the human hair cycle: an immunohistochemical study. Pigment Cell Res $2000 ; 13: 253-9$.

35. Commo S, Gaillard O, Bernard BA. Human hair greying is linked to a specific depletion of hair follicle melanocytes affecting both the bulb and the outer root sheath. BrJ Dermatol 2004 ; 150 : 435-43.

36. Commo S, Gaillard 0, Thibaut S, Bernard BA. Absence of TRP-2 in melanogenic melanocytes of human hair. Pigment Cell Res 2004 ; $17: 488-97$.

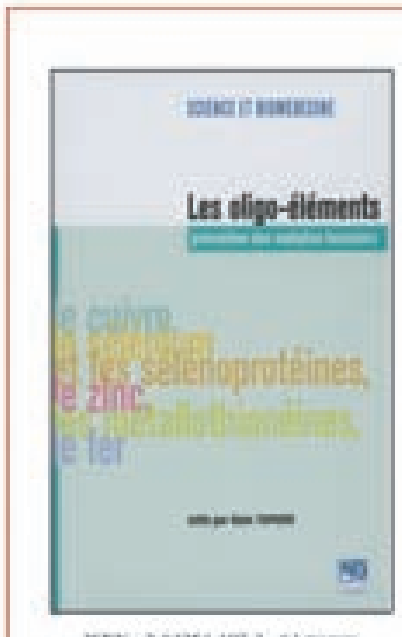

Bon de commande

A retourner a FDK, 10 Villa d'Orḱans - 75014 PARIS

Ta. : 0153910606 - Fax : 1153910607 - E-mail : editerial edk-fr

NOM :

Prtacen:

Adresse :

Code postal :

Ville

Pays:

Fonction :

Je soutaine recevoir rownąe Les oligo-eléments : $10 \ell+3 \ell$ de port $=13$ € TTC

7 Par chedyec, a rendre de E D K

7 Per cane losowe :

Carte n"

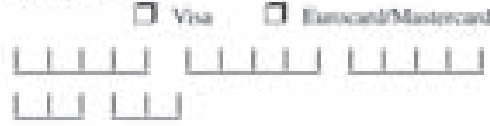

Signaure:

Date d'expineson

TIRÉS À PART

B.A. Bernard

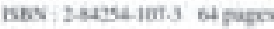

